\title{
Enhanced Thermal-to-Flexible Phase Change Materials Based on Cellulose/Modified Graphene Composites for Thermal Management of Solar Energy
}

Yongqiang Qian ${ }^{1,2,3}$, Na Han 1,2,3,4*, Zongxuan Zhang, 2, 3, Ruirui Cao ${ }^{1,2,3}$, Linli $\operatorname{Tan}^{1,2,3}$, Wei $\mathrm{Li}^{1,2,3}$, Xingxiang Zhang ${ }^{1,2,3 *}$

${ }^{1}$ State Key Laboratory of Separation Membranes and Membrane Processes, Tianjin 300387, China

${ }^{2}$ Tianjin Municipal Key Lab of Advanced Fiber and Energy Storage Technology, Tianjin 300387, China

${ }^{3}$ School of Material Science and Engineering, Tiangong University, Tianjin 300387, China

${ }^{4}$ Department of Textile Engineering, Chemistry \& Science, College of Textiles, North Carolina State University, Raleigh, North Carolina 27695, United States

\section{Corresponding Author}

*E-mail: hannatjpu@gmail.com; zhangpolyu@aliyun.com. 


\section{Preparation of initiator-modified cellulose 2-bromopropionylate (Cell-Br)}

Cellulose (1.25 g, $7.72 \mathrm{mmol})$ was suspended in $24 \mathrm{~mL}$ of anhydrous DMAc and stirred at $120^{\circ} \mathrm{C}$ for $1 \mathrm{~h}$. The slurry was then cooled to $100{ }^{\circ} \mathrm{C}$, and $\mathrm{LiCl}(3 \mathrm{~g}, 70.8$ mmol) was added and stirred for another $30 \mathrm{~min}$. The mixture was cooled to room temperature and kept stable overnight. BPB $(3.5 \mathrm{~g}, 38.7 \mathrm{mmol})$ was dropwised to the homogeneous solution in an ice/water bath. When the dropping process was completed, the reaction mixture was left to room temperature. After $4 \mathrm{~h}$, the solution was heated to $50{ }^{\circ} \mathrm{C}$ and stirred for $2 \mathrm{~h}$. The reaction mixture was allowed to cool to room temperature, and the polymer was isolated by precipitation in ethanol. Further purification was achieved by the re-dissolution of the crude product in $60 \mathrm{~mL}$ of acetone and re-precipitation in ethanol to produce a white powder. The resulting product was dried in vacuum at $50{ }^{\circ} \mathrm{C}$. The obtained yield is $72 \mathrm{wt} . \%$.

a

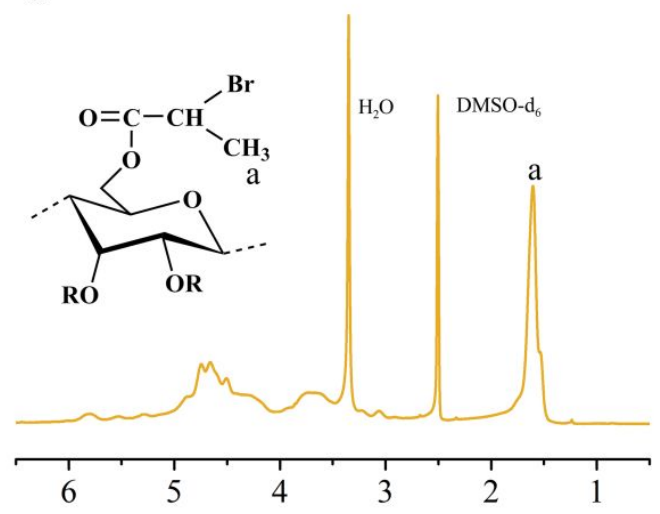

b

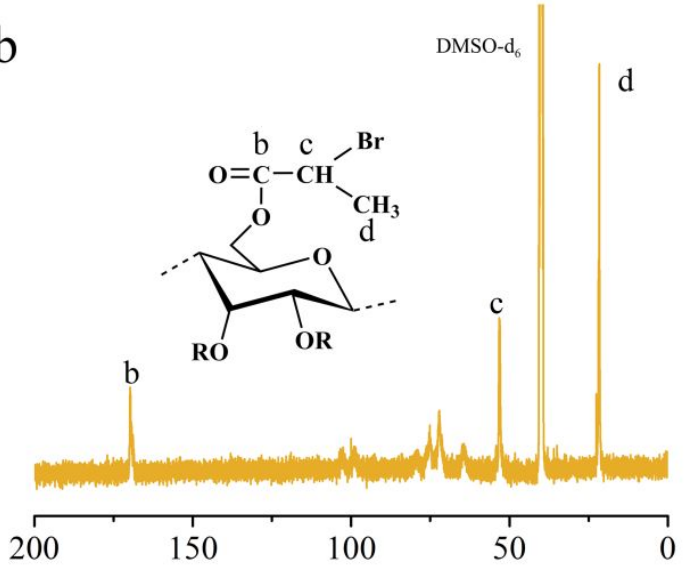

Figure S1. (a) ${ }^{1} \mathrm{H}$ NMR and (b) ${ }^{13} \mathrm{C}$ NMR spectra of Cell-Br.

The resulting ${ }^{1} \mathrm{H}$ NMR spectrum (Figure S1a) shows a peak at $\delta=1.59 \mathrm{ppm}$ that is attributed to the methyl protons of the bromopropionyl group in Cell-Br, with a substitution degree of 1.75 . Meanwhile, the chemical shift at $170 \mathrm{ppm}$ in the ${ }^{13} \mathrm{C}$ NMR spectrum (Figure S1b) confirms the presence of the carbonyl group. The peaks 
corresponding to methyl and methenyl carbon atoms appear at 22 and $53 \mathrm{ppm}$, respectively. However, a comparison of the FTIR spectra of cellulose and Cell-Br (Figure 2d) clearly shows that the intensity of the hydroxyl group band in the former decreases upon reaction with 2-bromopropionyl bromide. This is probably due to the substitution of some cellulosic hydroxyl groups by propionyl groups. The new peaks appearing at $1752 \mathrm{~cm}^{-1}$ and $703 \mathrm{~cm}^{-1}$ in the Cell-Br FT-IR spectrum are attributed to the carbonyl and bromo groups, respectively. Overall, the spectroscopic analyses confirm the successful preparation of the initiator-modified cellulose, Cell-Br.

\section{Preparation of modified GN nanosheets (GN16)}

Modified GN, denoted by GN16, was synthetized according to the following procedure. First, $1 \mathrm{~g}$ of GN was dispersed in DMAc by ultrasonication for $2 \mathrm{~h}$. Subsequently, HQ inhibitor was added to the uniform suspension, followed by the slow dropping of $15 \mathrm{~g}$ of A16 (50 mmol). The solution was continually mixed at $180^{\circ} \mathrm{C}$ under nitrogen atmosphere. $12 \mathrm{~h}$ later, the GN16 product was filtered and rinsed extensively with tetrahydrofuran (THF), then it was dried and stored for subsequent use.

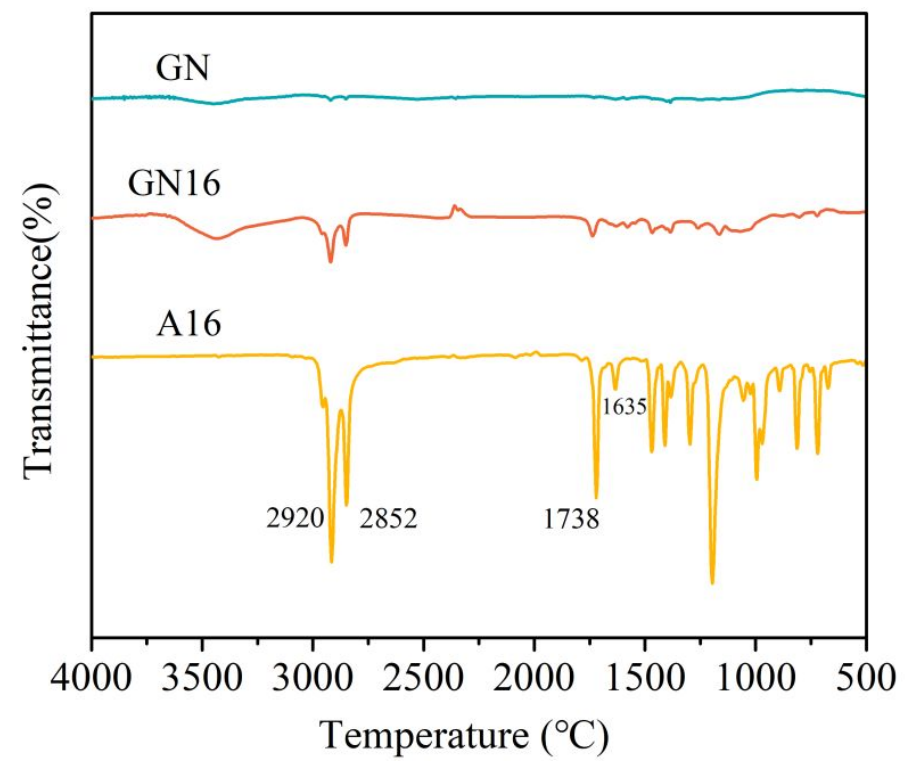


Figure S2. FT-IR spectra of GN, A16 and GN16
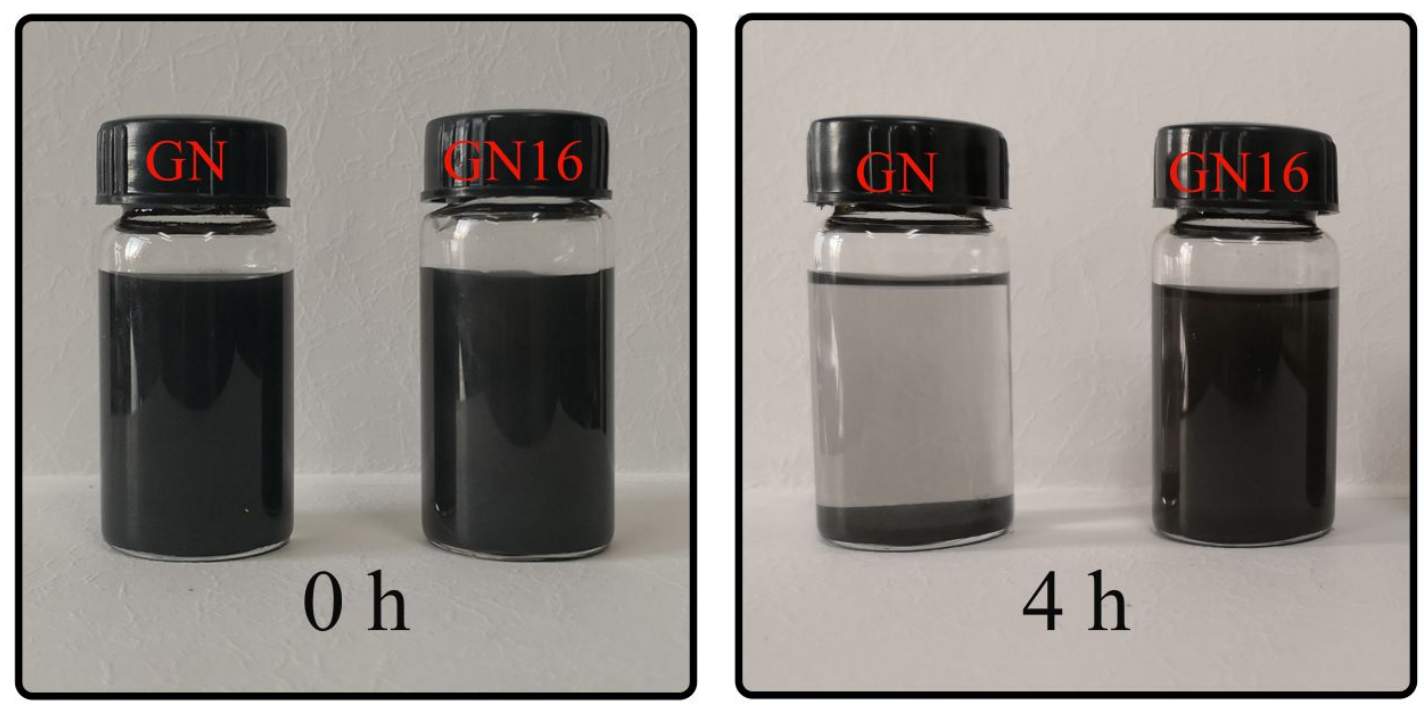

Figure S3 The dispersibility of GN and GN16 in DMAc after 4 h standing.
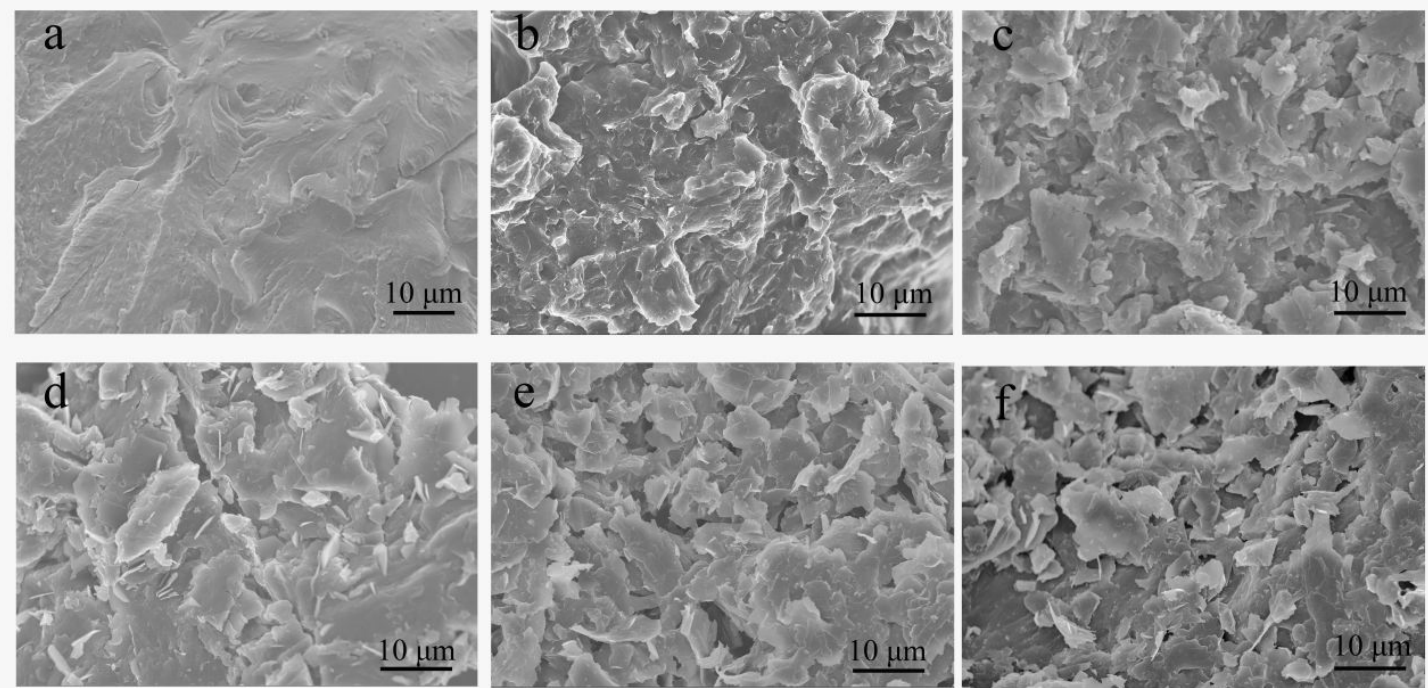

Figure S4. SEM images of CP (a), CPmG-1 (b), CPmG-3 (c), CPmG-5 (d), CPmG-7

(e) and CPmG-9 (f). 
Table S1. X-ray diffractometry data

\begin{tabular}{|c|c|c|c|c|}
\hline \multirow{2}{*}{ Sample } & \multicolumn{2}{|c|}{$2 \theta$} & \multirow{2}{*}{$\begin{array}{c}\mathrm{d}_{110} \\
(\mathrm{~nm})\end{array}$} & \multirow{2}{*}{$\begin{array}{c}\mathrm{d}_{002} \\
(\mathrm{~nm})\end{array}$} \\
\hline & 110 & 002 & & \\
\hline GN & - & 26.48 & - & 0.336 \\
\hline GN16 & 21.62 & 26.68 & 0.410 & 0.334 \\
\hline PA16 & 21.50 & - & 0.412 & - \\
\hline $\mathrm{CP}$ & 21.35 & - & 0.415 & - \\
\hline CPmG-1 & 21.44 & 26.50 & 0.413 & 0.336 \\
\hline CPmG-3 & 21.46 & 26.54 & 0.413 & 0.336 \\
\hline CPmG-5 & 21.45 & 26.62 & 0.413 & 0.334 \\
\hline CPmG-7 & 21.44 & 26.74 & 0.413 & 0.332 \\
\hline CPmG-9 & 21.40 & 26.50 & 0.414 & 0.336 \\
\hline
\end{tabular}

Table S2. Crystalline properties of PA16 and CPmG-x from DSC analysis

\begin{tabular}{ccccc}
\hline Specimen & $\begin{array}{c}\mathrm{GN} 16 \\
(\%)\end{array}$ & $\begin{array}{c}\Delta \mathrm{H}_{\mathrm{m}} \\
(\mathrm{J} / \mathrm{g})\end{array}$ & $X_{c}(\%)$ & $n_{c}$ \\
\hline PA16 & - & 93 & 32.8 & 6.9 \\
CP & 0 & 77 & 27.2 & 5.7 \\
CPmG-1 & 1 & 87 & 30.7 & 6.5 \\
CPmG-3 & 3 & 96 & 33.9 & 7.2 \\
CPmG-5 & 5 & 103 & 36.3 & 7.7 \\
CPmG-7 & 7 & 90 & 31.7 & 6.7 \\
CPmG-9 & 9 & 78 & 27.3 & 5.8 \\
\hline
\end{tabular}

The crystallinity $\left(X_{c}\right)$ of the synthesized SSPCMs and the number of crystallized $\mathrm{CH}_{2}$ and $\mathrm{CH}_{3}$ groups $\left(n_{c}\right)$ in the side chains of PA16, CP, and CPmG-x materials were calculated according to equations (S1) and (S2), respectively:

$X_{C}=\frac{\Delta \mathrm{H}_{\mathrm{m}}(\mathrm{n}) \times 14.026}{\mathrm{C}+\mathrm{k}(\mathrm{n})}$ 
$n_{c}=\frac{\Delta \mathrm{H}_{\mathrm{m}} \times \mathrm{M}_{\mathrm{unit}}}{\mathrm{k}}$

Where $\mathrm{n}$ is the number of methylene and methyl groups in the side chains, and $\mathrm{M}_{\text {unit }}$ is the relative molecular mass of the structural unit of the phase change working substance $\left(296.49 \mathrm{~g} \mathrm{~mol}^{-1}\right) .{ }^{1} \mathrm{C}$ and $\mathrm{k}$ values are zero and $3971 \mathrm{~J} \mathrm{~mol}^{-1}$, respectively. ${ }^{2,3}$

The calculated values listed in Table S2 show that the macro-fluidity of PA16 is greatly impeded upon grafting onto the cellulose support due to the restriction of the free movement of side chains, even at temperatures higher than the melting point. Accordingly, the $X_{c}$ and $n_{c}$ of $\mathrm{CP}$ are significantly less than the corresponding values of PA16. However, $X_{c}$ and $n_{c}$ are gradually enhanced upon the incorporation of up to 5\% GN16 into CP. The $X_{c}$ of material containing 5\% dispersed GN16 is greater than both, the CP (9.1\% greater) and pure PA16 values, which signifies that the incorporation of GN16 into the material increases the number of $\mathrm{CH}_{2}$ groups in the crystal lattice. Therefore, it may be concluded that GN16 promotes alkyl chain crystallization, resulting in higher $n_{c}$ values. Increasing the GN16 content beyond 5\% results in the gradual decrease of $X_{c}$ and $n_{c}$ values in CPmG-x. This is due to the restriction of side chain free movement by excessive GN16, which ultimately leads to reduced enthalpy of the material. 
Table S3. Thermal stabilities of GN, GN16, Cell-Br, CP and CPmG-X

\begin{tabular}{cccc}
\hline Sample No. & Ta do $\left({ }^{\circ} \mathrm{C}\right)$ & $\mathrm{Tb} d p\left({ }^{\circ} \mathrm{C}\right)$ & Mass loss $(\%)$ \\
\hline GN & - & - & 0 \\
GN16 & 341 & 369 & 15 \\
Cell-Br & 277 & 290 & 85 \\
CP & 299 & 407 & 96 \\
CPmG-1 & 376 & 408 & 92 \\
CPmG-3 & 375 & 408 & 91 \\
CPmG-5 & 370 & 406 & 90 \\
CPmG-7 & 372 & 406 & 89 \\
CPmG-9 & 368 & 406 & 87 \\
\hline
\end{tabular}

a: The onset decomposition temperature; ${ }^{b}$ : The fastest decomposition temperature.

\section{Shape stabilization performance and thermal reliability behavior}

Shape-stabilized test of SSPCMs was realized by visual observation method. For direct observation, the pressed PA16, CP, and CPmG-x with a diameter $20 \mathrm{~mm}$ and $7.5 \mathrm{~mm}$ in thickness was put into a $60^{\circ} \mathrm{C}$ oven for $30 \mathrm{~min}$, and then recorded the changes of disc size and morphology. Additionally, Thermal reliability of 100 heating/cooling cycles was conducted by a programmable controller (Giant Force) with temperature changing from 0 to $100^{\circ} \mathrm{C}$. After that, the thermal reliability and the structural stability were determined by DSC and FT-IR analysis before and after 100 heating/cooling cycles. 


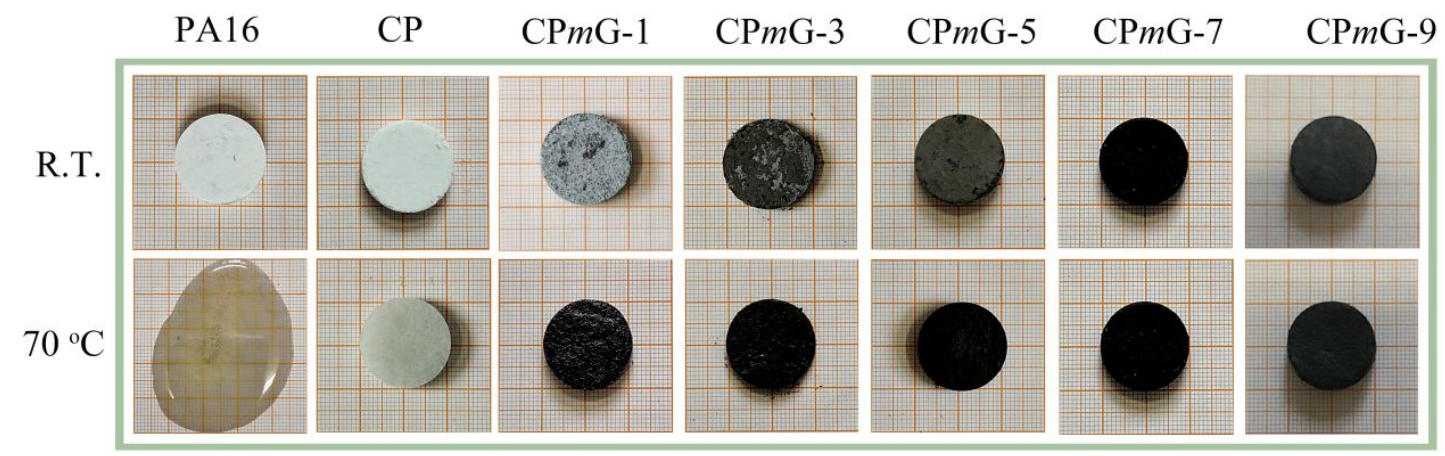

Figure S5. Shape-stability of PA16, CP and CPmG-x with respect to temperature through the visual observation

The leakage and flowability of solid-liquid PCMs in melting state is the biggest barrier for practical applications. Here, leakage tests for CPmG-x are performed in hot stage at $70^{\circ} \mathrm{C}$ for $10 \mathrm{~min}$. The thermal behavior of the thermo-responsive samples is observed by a digital camera as shown in Figure S3. For CP, it is a white and opaque wafer due to the crystalline of PA16 at ambient temperature. The sample CP just become semitransparent and changes to an elastomer without any leakage after 10 min heating, implying the melting of side chain PA16 crystals. All thermo-responsive samples CPmG-x are black one for due to the introduction of the GN16. There is no significant change at the macroscopic level and they remained in the solid state. It is proved that the all thermo-responsive CPmG-x composites are the solid-solid phase change materials and have possess outstanding shape stability, implying the melting of PA16 crystals. 

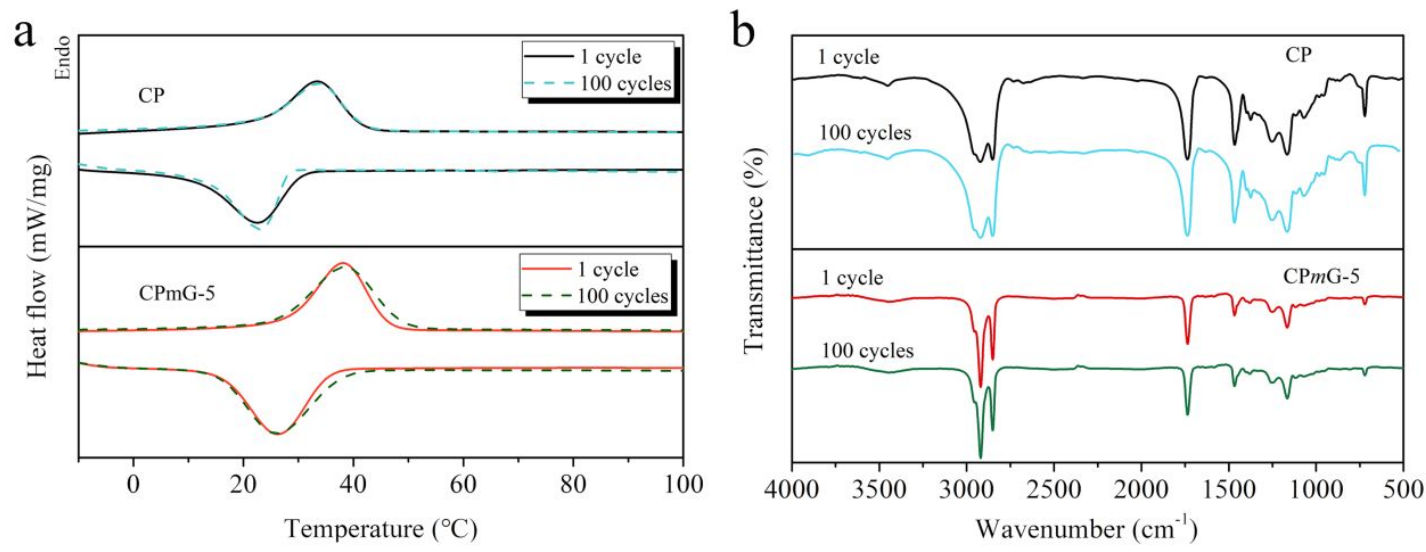

Figure S6. DSC curves (a) and FT-IR spectra (b) of CP and CPmG-5 before and after the 100 thermal cycling treatments.

Thermal reliability and cycling stability of PCMs are crucial points in the area of thermal energy storage (TES) application. Here, taking $\mathrm{CP}$ and $\mathrm{CPmG}-\mathrm{x}$ as an example, the thermal reliability and structural stability are compared before and after 100 times thermal cycles. The DSC and FT-IR results are showed in Figure S4a and b, and the corresponding thermal data is summarized in Table 2. After 100 times thermal cycles, $\mathrm{T}_{\mathrm{m}}$ and $\mathrm{T}_{\mathrm{c}}$ of $\mathrm{CP}$ and $\mathrm{CPmG}-5$ exhibit a $0.3-0.8^{\circ} \mathrm{C}$ downshift as compared with the ones untreated. Meanwhile, the phase transition enthalpy of $\mathrm{CP}$ and CPmG-5 shows a 1-3 J/g decrement, which is negligible for thermal energy storage applications. In Figure S4b, it is shown that there is no obvious change in the structure of the samples after 100 thermal cycles. It is further demonstrated that the SSPCMs have a good structural stability. It could be concluded that the CPmG-x composites can be a good alternative in the area of TES materials. 
Table S4. TC of relative polymer composites doped with GN nanofillers in the available literature

\begin{tabular}{|c|c|c|c|c|c|}
\hline \multirow{2}{*}{ Reference } & \multirow{2}{*}{ Sample } & \multicolumn{2}{|c|}{ Dimensions } & \multirow{2}{*}{$\begin{array}{c}\text { Filter } \\
\text { content } \\
(\%)\end{array}$} & \multirow{2}{*}{$\begin{array}{c}\mathrm{TC} \\
(\mathrm{W} / \mathrm{mK})\end{array}$} \\
\hline & & Size & Thick & & \\
\hline 14 & Paraffin/GN & \multicolumn{2}{|c|}{$\mathrm{N} / \mathrm{A}$} & 10 & 0.5 \\
\hline 16 & $\begin{array}{l}\text { Paraffin } \\
\text { /xGnP-1 }\end{array}$ & $\sim 10 \mu \mathrm{m}$ & $\sim 10 \mathrm{~nm}$ & 5 & 0.5 \\
\hline \multirow{2}{*}{22} & \multirow{2}{*}{ Paraffin/xGnP } & \multirow{2}{*}{$\sim 15 \mu \mathrm{m}$} & \multirow{2}{*}{$\sim 10 \mathrm{~nm}$} & 3 & 0.454 \\
\hline & & & & 5 & 0.616 \\
\hline \multirow{2}{*}{24} & LA/GO foam & \multirow{2}{*}{\multicolumn{2}{|c|}{ N/A }} & & 0.91 \\
\hline & LA/GO-LA & & & 5 & 127 \\
\hline \multirow{6}{*}{40} & & \multirow{6}{*}{$3.9 \mu \mathrm{m}$} & \multirow{6}{*}{$\begin{array}{c}\sim 100 \\
\mathrm{~nm}\end{array}$} & 1.1 & 0.3 \\
\hline & Epoxy/GNPs & & & 2.2 & 0.32 \\
\hline & & & & 4.5 & 0.5 \\
\hline & Epoxy/ & & & 1.1 & 0.3 \\
\hline & modified & & & 2.2 & 0.43 \\
\hline & GNPs & & & 4.5 & 1.25 \\
\hline \multirow{5}{*}{ Our work } & CPmG-1 & \multirow{5}{*}{$\sim 10 \mu \mathrm{m}$} & \multirow{5}{*}{$3-9 \mathrm{~nm}$} & 1 & 0.3 \\
\hline & CPmG-3 & & & 3 & 0.49 \\
\hline & CPmG-5 & & & 5 & 0.7 \\
\hline & CPmG-7 & & & 7 & 0.95 \\
\hline & CPmG-9 & & & 9 & 1.32 \\
\hline
\end{tabular}

\section{REFERENCE}

(1) Li, J.; Wang, H.-X.; Kong, L.; Zhou, Y.; Li, S.-Q.; Shi, H.-F., Phase transition and side-chain crystallization of poly(methyl vinyl ether-alt-maleic anhydride)-g-alkyl alcohol comb-like polymers. Macromolecules 2018, 51, 8922-8931.

(2) Jordan, E.-F.; Feldeisen, D.-W.; Wrigley, A.-N., Side-chain crystallinity. I. heats of fusion and melting transitions on selected homopolymers having long side chains. J. Polym. Sci. Part A-1 1997, 9, 1853-1852.

(3) Jin, Y.-M.; Liu, H.-H.; Wang, N.; Hou, L.-C.; Zhang, X.-X., Dispersibility and S-10 
chemical bonds between multi-walled carbon nanotubes and poly(ether ether ketone) in nanocomposite fibers. Mater. Chem. Phys. 2012, 135 (2-3), 948-956. 